\title{
Numerical Study of Turbulent Mixed Convection Heat Transfer in a Semi-Circle Cavity Contains a Rotating Cylinder
}

\author{
Falah A. Abood ${ }^{1} \quad$ MujtabaAlmudhaffar ${ }^{2} \quad$ Zinah S. Dawood $^{2}$ \\ 1.Mechanical Engineering Department/Engineering College, University of Basrah, Iraq \\ 2.Basrah Engineering Technical College/ Southern Technical University-Basra-Iraq
}

\begin{abstract}
The investigation of mixed convection inside the semi- circle cavity with a fixed cylinder and rotating has been performed numerically. The bottom wall was maintained to constant heat flux while the curved wall was subjected to ambient and an adiabatic rotating cylinder presence in center cavity. The computation fluid dynamics (CFD)modeling includes numerical solutions of the conservation equation for mass ,momentum and energy, numerical prediction by using ansys FLUENT version 15. The finite volume method were used for solving governing differential equations and the turbulence model was applied SST(K- $\omega)$. The results were represented of streamlines, isotherm counters and distribution local Nusselt number at Rayleigh number $2.9 \times 10^{8}$ identical to heat flux $\mathrm{q}=290 \mathrm{w} / \mathrm{m}^{2}$ and angular velocity of cylinder $\omega=0,62.8,83.73$ and $89.5 \mathrm{rad} / \mathrm{s}$ clockwise and inclination angle of cavity $0^{\circ}, 30^{\circ}$ and $90^{\circ}$. The result shows that the effect of both rotating cylinder, inclination angle led to enhancement heat transfer.
\end{abstract}

Keywords: Mixed convection, turbulence model, inclination angle, semicircle cavity.

DOI: $10.7176 / \mathrm{JNSR} / 9-18-02$

Publication date:September $30^{\text {th }} 2019$

\section{Introduction}

The matter of mixed convection in the cavity is important both theoretically and experimentally. The mixed convection in cavities have immense engineering and industrial applications such as cooling electronic components, makings solar collectors and building insulation [1].There are researchers investigated the influences of convective flows in cavities, channels through the use of experimental and numerical procedures that attentive the impact the rotating body within cavity or without rotating. Billah et al.[2]investigated heat transfer for heated circular hollow cylinder within rectangular cavity. They found the important parameter for heat transfer, fluid flow and temperature distribution is position of the circular heated hollow cylinder.also ,found . the located of the hollow cylinder will be influenced on the heat transfer and fluid flow.Alam et al. [3] introduced that the existence of a rotating cylinder which impact the heat transfer characteristics within heated cavity. The results showed that rotation speeds and cylinder size will affect the flow field, temperature distribution and heat transfer rate . whilethe influence of conductivity ratio is not very prominent. Karimi et al.[4] investigated the effect mixed convection in a square cavity with the heated horizontal cylinders which existed midst of the cavity height .The results observed that heat transfer rates from heated cylinders and fluid temperature in the cavity will increase with increasing number of Richardson number and cylinders diameter .Park et al. [5] examined numerically in the two dimension square enclosure contains cold and hot cylinders concluded that increases in the number of Nusselt occurred when the cylinder surfaces and cavities were close together Ray and Chatterjee [6] examined numerically the heat transfer in lid-driven cavity which containing horizontal circular cylinder.The authors found the inside rotating cylinder causes a great increase in the heat transfer convection. Tahseen Alhattabetal.[7]introduced heating element was placed at various positions above the inclined wall of triangle cavity where Raylei number was $10^{5}$, $10^{6}, 10^{7}$ and $10^{8}$.There were Three various status normal, side and inverse of the triangular cavity.The results illustrated that for normal status of the cavity and when the heating element is at situated $0.2 \mathrm{~L}$ to $0.3 \mathrm{~L}$ from the edge of the triangle cavity causes a maximum $\Psi$ and high rate of heat transfer. Mehdi and Mushatt [8], studied numerically the effect heat transfer and fluid flow of the two dimensional within inclined square enclosure. The results presented shows that the rate of heat transfer was increased with the increase of Rayleigh number and decreased with the increase of angle of inclination from $0^{\circ} \leq \theta \leq 90^{\circ}$.singh and Bhargava, [9] Numerically studied the effect convection in awavy wall enclosure. They show that, the heat trans ferrate was highly depends on Rayleigh number ,Prandtl number andthe shape of the heat transfer surface because asthe wavy surface conveys the heat higher from the smooth surface. .Casado et al .[10] Numerically present was the new correlations to compute the impact heat transfer coefficients in enclosures in building. The second correlations used for turbulent system when $\mathrm{Ra}>106$ and another in laminar system when $\mathrm{Ra}<106$.Furthermore, for a vertical wall two new correlations had been done in turbulent system when $(107<\mathrm{Ra}<1011)$ and other in laminar system when $(\mathrm{Ra}<107)$. the results show that in the laminar flow $i$ the Nusselt number depends on the aspect ratio while the is un dependend on the aspect ratio in turbulent flow . The present study had been carried out on turbulent mixed convection in a semi circle cavity filled with air. The current study will be conducted numerically using a precise numerical procedure, and the results will be presented utilizing streamlines, isotherms. 


\section{Nomenclature}

\section{g gravitational acceleration}

$\mathrm{Cp}$ specific heat at constant pressure

q constant heat flux

$\mathrm{k}$ thermal conductivity

$\mathrm{Nu}_{\mathrm{L}}$ local Nusselt number

Pr Prandtl number

Ra Rayleigh number

$\mathrm{L}$ width of enclosure

$\mathrm{N}$ number of revolution per mint

$\mathrm{D}_{\mathrm{h}}$ hydraulic diameter

Greek symbol

$\mu$ dynamic viscosity

$\alpha$ thermal expansion cofficient

$\varnothing$ inclination angle

$\omega$ angular velocity

$\beta$ coefficient of volumetric expansion

$\mathrm{K}$ Turbulent kinetic energy

$\omega$ specific dissipation

$\rho$ density

Subscripts

adi adiabatic surface

f mean(bulk)temperature of fluid

L local

\section{Theoretical Formulations}

The present problem involves a semi-circle cavity contains the solid cylinder rotating at angular velocity as depicted in Fig. 1 . The cavity $\mathrm{R}=15 \mathrm{~cm}$ and with depth $\mathrm{Z}=75 \mathrm{~cm}$. The rotating cylinder with $\mathrm{D}=5 \mathrm{~cm}$ is located in the center of the cavity and it is rotate with clock wise direction. The cavity is heated from bottom by heater at constant heat flux. the external curved wall is exposed to the ambient temperature .The numerical model is a three dimensional semi- circle cavity filled with air. The flow is turbulent, steady and incompressible. The physical properties of the fluid are unaltered except for the density of the fluid which depends on the temperature. The angular velocity uses $\omega=062.8,83.73$ and $89.5 \mathrm{rad} / \mathrm{s}$. The Rayleigh number is theoretically examined $2.9^{*} 10^{8}$ and inclination angle $0^{\circ}, 30^{\circ}$ and $90^{\circ}$.

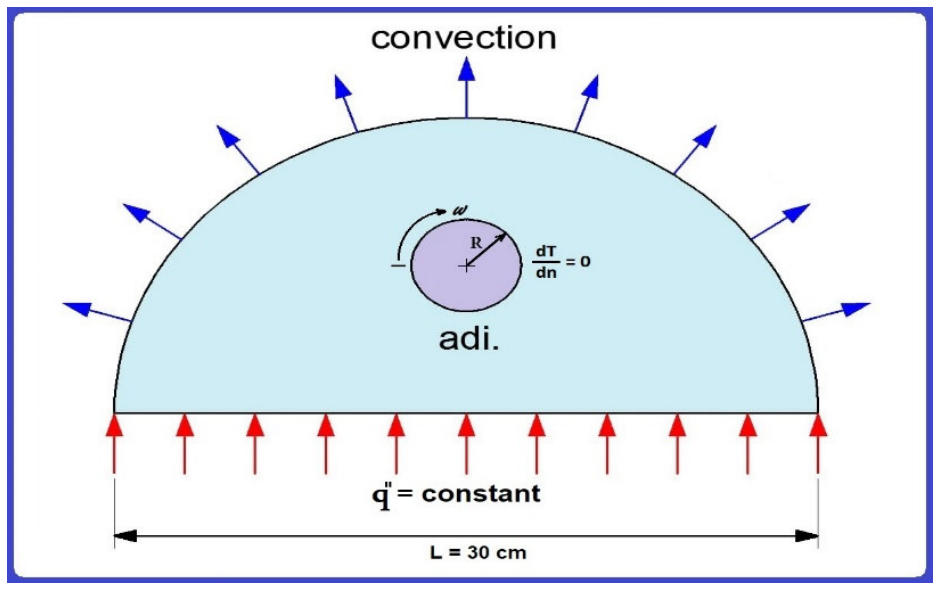

Continuity equation

$\frac{\partial}{\partial x}(\rho \bar{u})+\frac{\partial}{\partial y}(\rho \bar{v})+\frac{\partial}{\partial z}(\rho \bar{w})=0$

$\mathrm{x}$-Momentumequation 


$$
\frac{\partial}{\partial x}(\rho \bar{u} \bar{u})+\frac{\partial}{\partial y}(\rho \bar{u} \bar{v})+\frac{\partial}{\partial z}(\rho \overline{u w})=-\frac{\partial \bar{p}}{\partial x}+\left(\mu+\mu_{t)}\left(\frac{\partial^{2} \bar{u}}{\partial x^{2}}+\frac{\partial^{2} \bar{u}}{\partial y^{2}}+\frac{\partial^{2} \bar{u}}{\partial z^{2}}\right)\right.
$$

$\mathrm{y}$ - Momentum equation

$$
\frac{\partial}{\partial x}(\rho \bar{u} \bar{v})+\frac{\partial}{\partial y}(\rho \bar{v} \bar{v})+\frac{\partial}{\partial z}(\rho \bar{w} \bar{v})=-\frac{\partial \bar{p}}{\partial y}+\left(\mu+\mu_{t}\right)\left(\frac{\partial^{2} \bar{v}}{\partial x^{2}}+\frac{\partial^{2} \bar{v}}{\partial y^{2}}+\frac{\partial^{2} \bar{v}}{\partial z^{2}}\right)-\rho g \beta\left(\bar{T}_{\infty}-\bar{T}\right)
$$

z-momentum equation

$$
\frac{\partial}{\partial x}(\rho \overline{u w})+\frac{\partial}{\partial y}(\rho \bar{v} \bar{w})+\frac{\partial}{\partial z}(\rho \bar{w} \bar{w})=-\frac{\partial \bar{p}}{\partial z}+\left(\mu+\mu_{t}\right)\left(\frac{\partial^{2} \bar{w}}{\partial x^{2}}+\frac{\partial^{2} \bar{w}}{\partial y^{2}}+\frac{\partial^{2} \bar{w}}{\partial z^{2}}\right)
$$

Energy equation

$$
\frac{\partial}{\partial x}\left(\rho \bar{u} c_{p} \bar{T}\right)+\frac{\partial}{\partial y}\left(\rho \bar{v} c_{p} \bar{T}\right)+\frac{\partial}{\partial z}\left(\rho \bar{w} c_{p} \bar{T}\right)=K\left(\frac{\partial^{2} \bar{T}}{\partial x^{2}}+\frac{\partial^{2} \bar{T}}{\partial y^{2}}+\frac{\partial^{2} \bar{T}}{\partial z^{2}}\right)-\frac{\partial}{\partial x_{i}}\left(\frac{\partial \bar{T}}{\partial x_{i}} \frac{c_{p} \mu_{t}}{\sigma_{t}}\right)+q^{\prime \prime \prime}
$$

The governing equation of SST k- $\omega$ model for steady state turbulent flow are below.

$$
\begin{aligned}
& \frac{\partial}{\partial x_{i}}\left(\rho k u_{i}\right)=\frac{\partial}{\partial x_{j}}\left[\left(\mu+\frac{\mu_{t}}{\sigma_{k}}\right) \frac{\partial k}{\partial x_{j}}\right]+G_{k}-Y_{K}-S_{k} \\
& \frac{\partial}{\partial x_{i}}\left(\rho \omega u_{i}\right)=\frac{\partial}{\partial x_{j}}\left[\left(\mu+\frac{\mu_{t}}{\sigma_{\omega}}\right) \frac{\partial \omega}{\partial x_{j}}\right]+G_{\omega}-Y_{\omega}+D_{\omega}+S_{\omega}
\end{aligned}
$$

where $G_{k}$ represents the generation of turbulent kinetic energy the arises due to mean velocity gradients, $G_{\omega}$ is generation of $\omega$, and $Y_{k}$ and $Y_{\omega}$ represent the dissipation of $\mathrm{k}$ and $\omega$ due to turbulence. $\alpha_{k}$ and $\alpha_{\omega}$ are the turbulent Prandtl numbers for $\mathrm{k}$ and $\omega . S_{\mathrm{k}}$ and $S_{\omega}$ are source terms defined by the user. The term for the dissipation of $\mathrm{k}$ due to turbulence, $Y_{k}$ is defined as:

$Y_{k}=\rho \beta^{*} f_{\beta}{ }^{*} k \omega$

and the term for the dissipation of $\omega$ due to turbulence, $\mathrm{Y}_{\omega}$ is defined as:

$Y_{\omega}=\rho \beta f_{\beta} \omega^{2}$

The turbulent viscosityiscomputedas:

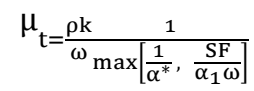

where $\mathrm{S}$ is the strain rate magnitude and $\alpha^{*}$ is the damping coefficient.Blendingfunctions have been added to the SST model to ensure that the model equations behaveappropriately in both near wall and far field zones

$$
\begin{aligned}
& \sigma_{\mathrm{k}}=\frac{1}{\mathrm{~F}_{1} / \sigma_{\mathrm{k}, 1+}(1-\mathrm{F} 1) / \sigma_{\mathrm{k}, 2}} \\
& \sigma_{\omega=\frac{1}{F_{1} / \sigma_{\omega, 1+}(1-F 1) / \sigma_{\omega, 2}}}
\end{aligned}
$$

Where $F_{1}=\tanh \left(\Phi_{1}^{4}\right), F_{2}=\tanh \left(\Phi_{2}^{2}\right)$

The constants specific to the SST k-omega model aredefined as $\sigma_{\omega, 1}=2.0, \sigma_{\omega, 2}=1.168, \sigma_{k, 1}=1.176, \sigma_{k, 2}=1.0$, $a_{1}=0.31$.[11]

The angular velocity of the cylinder is calculated as follows:

$\omega=2 \pi \mathrm{N} / 60$

The heat flux along the heated surface can be calculated where V: is the voltage, I: is the current and A is the heated surface area[12] and the average Nusselt number as follow .

$$
\begin{aligned}
& q^{\prime \prime}=\frac{0.96 V I}{A} \\
& N u_{\text {av. }}={ }^{q "} D h\left(\mathrm{~T}_{S}-\mathrm{T}_{f}\right) K
\end{aligned}
$$

$\mathrm{Ra}=\mathrm{g} \beta \mathrm{q}^{\prime \prime} \mathrm{D}_{\mathrm{h}}{ }^{4} / \mathrm{kv \alpha}$ 


\section{Numerical Analysis}

Fluid flow and heat transfer problems can be solve by using Computational Fluid Dynamics (CFD) techniques .In this project CFD simulations depend on the finite volume method (FVM) that the uses ANSYSFLUENT version 15 for solving the continuity, momentum and energy equation. Analysis of simulation results. This included the analysis for the $0^{\circ}$ horizontal cavity, tilted cavity with $\emptyset=30^{\circ}$ and $90^{\circ}$ vertical cavity .Pressure-velocity coupling indicates to the numerical algorithm that utilizes a combination of continuity and momentum equations to derive an equation for pressure (or pressure correction). this coupling of pressure and velocity fields are carried out by using the coupled algorithm due to the convergence will be faster and that pressure correction equation and compute cell-face pressures by using PRESTO scheme .The converges criteriafor solution $10-^{4}$ for each continuity and Naiver stock equations and $10^{-8}$ for energy equation.

\section{Result and Discussion}

4.1 Temperature and Streamlines Counters

Temperature and stream function contours for the paradigms cases with angles $\varnothing=0^{\circ}, 30$ and $90^{\circ}$, Rayley number $2.9 \times 10^{8}$ correspondingly to heat flux $290 \mathrm{w} / \mathrm{m}^{2}$ and with angular velocity $\omega=0,62.8,83.73$ and $89.5 \mathrm{rad} / \mathrm{s}$ are shown in figures. Rayleigh number $2.9 * 10^{8}$ investigated numerically at angular velocity $0,62.8,83.73 \mathrm{and} 89.5 \mathrm{rad} / \mathrm{s}$. Fig.1shows the Investigation of the influence of free convection in the case without rotating the cylinder for heat flux $290 \mathrm{w} / \mathrm{m} 2$, the right side of this figure illustrate the streamlines in the horizontal cavity $\left(\varnothing=0^{\circ}\right)$, It was formed a pair of vortices each vortex is located beside the cylinder. The intensity of this vortices is reducing near the curved wall of cavity due to reduces the flow velocity. A pattern of a unicellular flow is noticed at each tilt angle also there is a small effect of tilt angles on the intensity of the streamlines. The rotation pattern in the cavity is special due to the buoyancy force is the controlling force on the flow. The buoyancy force resulting by gravity which corresponds to the buoyancy effect reinforce each other, so it is formed of a strong vortex, which occupy most space of the cavity.

Figure .2 illustrates The distribution of streamlines and temperature counters at heat Flux $290 \mathrm{w} / \mathrm{m} 2$ and angler velocity $62.8 \mathrm{rad} / \mathrm{s}$ with various inclination angles. The left side of the figure represents the temperature counter, while the right side represents the streamlines counter. From the distribution of temperature it can be noticed there is a gradient in the air temperature within the gap for each tilt angle and that the thermal layer covers all the area within the gap due to the effect of cylinder rotation. At the zero angle situation it can be observed the irregular distribution of temperatures on both sides of the rotary cylinder and that the maximum values of temperature is located in the region below the cylinder while the minimum temperature towards the right side which is the values of temperature higher than the left of the cylinder rotor, the reason for the difference in the distribution of temperature in those areas is that in the left side the direction of the flow is opposing with the buoyancy effect while the direction of the flow on the right side is aiding the buoyancy effect. In the $30^{\circ}$ angle it can be seen a uniform distribution of the temperature inside the gap on both sides of the cylinder and that the highest values must be in the confined area below the cylinder because of the different angle of inclination of the load currents with the flow direction. For angle $90 \mathrm{o}$ it can be note irregularity in the distribution of temperature within the gap and that the temperature at the top of the gap is higher than the bottom and this is is attributed to that the direction of the buoyancy effect is opposing the flow direction along the hot surface is and that the effect appears in the upper region of the cavity where less air density. The right side of Figure .2 shows the streamlines counter for various of tilt angles, it can be observed that two cells are formed within the gap due to the difference in pressure values.

At the $0^{\circ}$ angle it can be seen one cell in the region at the upper of the cylinder while the other was formed at the right of the cylinder, this occurs because the convection currents are opposing the flow direction that causes a pressure differences in this region. For tilt angle $30^{\circ}$, the vortices are in the region towards the right side of the cylinder and its intensity is higher than the angle in the case of zero angle because of the change the direction of the convection currents with the flow direction. For angle $90^{\circ}$ it can be noticed the regularity in the distribution of streamlines on both sides of the rotary cylinder near the hot surface this is due to the opposing of convection currents with the direction of the flow causing pressure differences in these areas and also note that the flow intensity here is slightly higher than the situation in the corner zero and less than the angle $30^{\circ}$.

The left side of the figure. 3 represents the temperature counter which indicates to the distribution of temperature within the cavity at heat flux $290 \mathrm{w} / \mathrm{m} 2$ and angular velocity equal $83.73 \mathrm{rad} / \mathrm{s}$ for two case $0^{\circ}, 30^{\circ}$, results show same profile because of the combination effect of gravity and buoyancy force . It can be noticed that the trend of the curves of tilt angle $60^{\circ}$ is different from the previous state. This behavior illustrates enough mixing inside closed cavity. For the tilt angle equal $90^{\circ}$ the results are illustrate uniform distribution.

The right side of figure. 3 shows the distribution of the streamlines for heat flux $290 \mathrm{w} / \mathrm{m} 2$ with the effect of the tilt angles $0^{\circ}, 30^{\circ}$ and $90^{\circ}$ on the flow field. For $\varnothing=0^{\circ}$, it can be seen two cells, one is located in the top and the other at down, the top vortex dominates on the most space of cavity and the bottom vortex is existed in the lower left area. It can be seen the increasing the impact of buoyancy force when is compared to the previous state . 
For $\emptyset=30^{\circ}$ there are a asymmetrical pair of vortex are formed, a small vortex was created in the upper right space of the cavity because of the inclination angle of convection current, the left vortex has a non-uniform form. For $\varnothing=90^{\circ}$ observes the two vortex one is existed on the top and the other at the bottom of the cylinder which are appear combined impact of rotating and inclination.

At the right side of figure. 4 indicates the distribution of the streamlines for heat flux $290 \mathrm{w} / \mathrm{m} 2$, angular velocity $=89.5 \mathrm{rad} / \mathrm{s}$ and various tilt angles. For $\emptyset=0^{\circ}$ the vortex is formed at the top part and its location in the right area of the cavity. For $\varnothing=30^{\circ}$ a pair of vortex appears on the left side of the cavity and the vortices appear to be approaching each other. At $\varnothing=90^{\circ}$ Obviously the streamlines contour distribution is similar to the previous situation of figure. 4 but Contains a high value of velocity values at the same flow of heat.

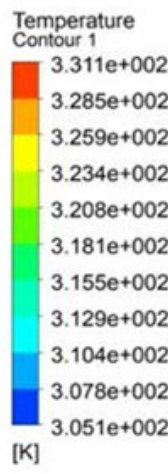

Temperature

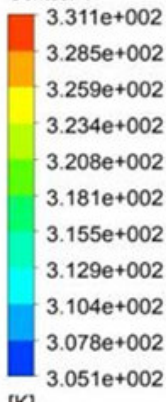

$[\mathrm{K}]$

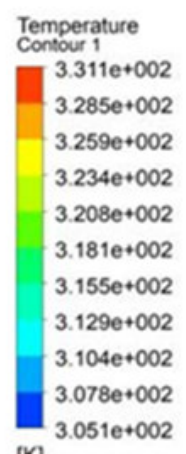

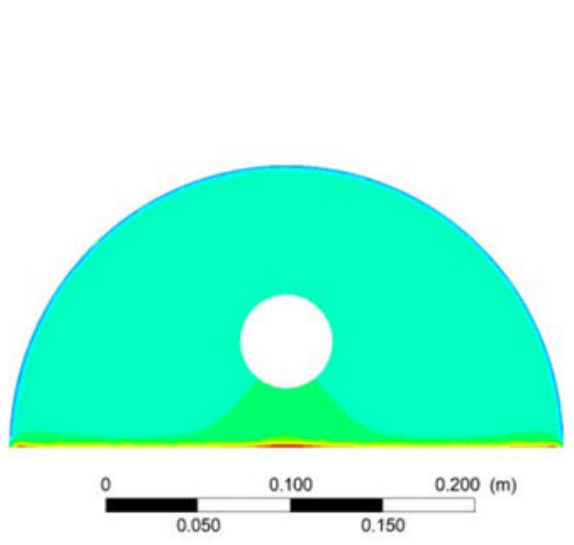

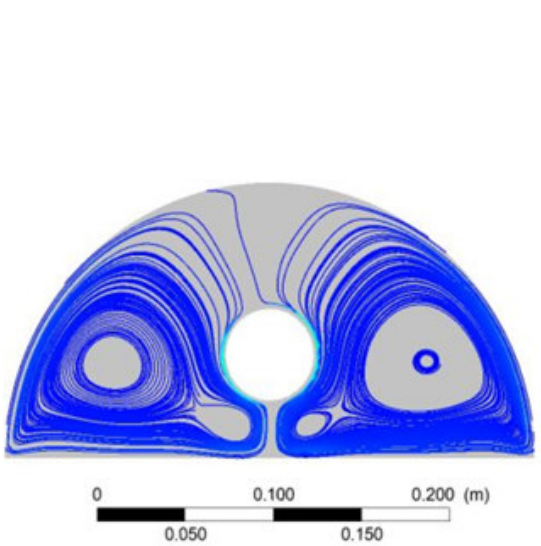

Velocity
Streamline 1

$6.955 \mathrm{e}-003$

$5.217 e-003$

$3.478 \mathrm{e}-003$

$1.739 \mathrm{e}-003$

$0.000 e+00 c$ [m s^-1]
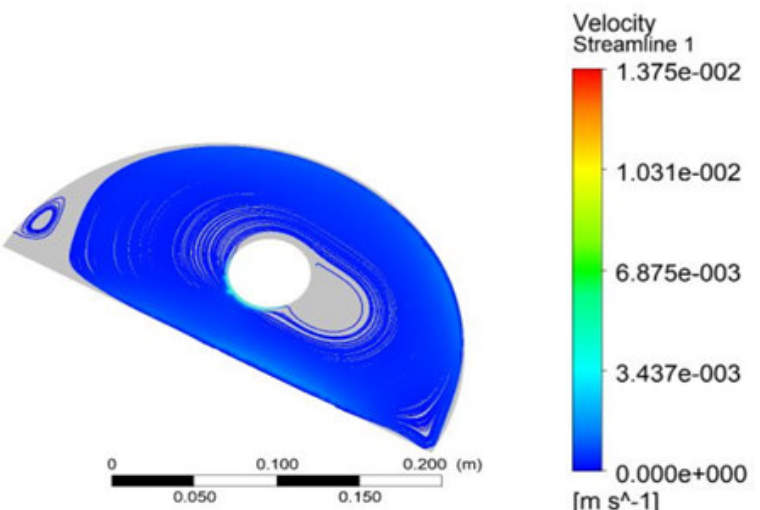

$\left[\mathrm{m} \mathrm{s}^{\wedge}-1\right]$
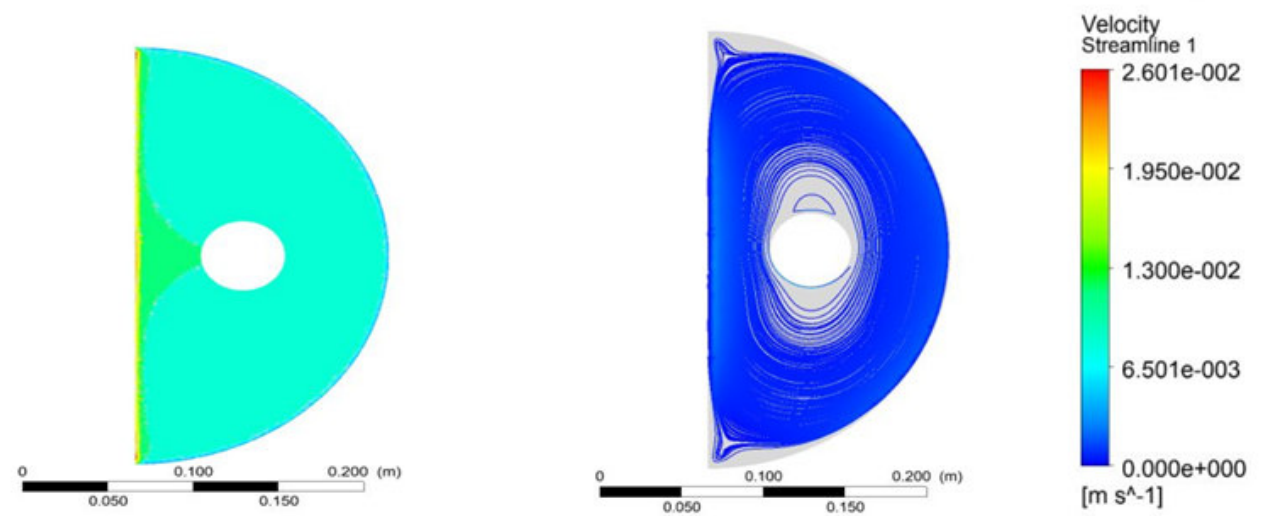

Fig.1.Temperature distribution and streamlines contours at $q^{\prime \prime}=290 \mathrm{w} / \mathrm{m} 2$ and with inclination angle $\varnothing^{\circ}=0^{\circ}, 30^{\circ}, 90^{\circ}$ respectively. 


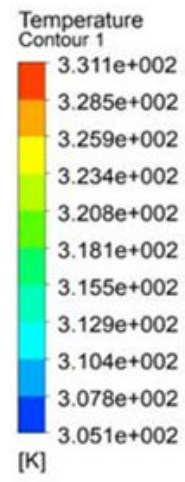

Temperature

$3.311 \mathrm{e}+002$

$3.285 \mathrm{e}+002$

$3.259 \mathrm{e}+002$

$3.234 \mathrm{e}+002$

$3.208 \mathrm{e}+002$

$3.181 \mathrm{e}+002$

$3.155 \mathrm{e}+002$

$3.129 \mathrm{e}+002$

$3.104 \mathrm{e}+002$

$3.078 \mathrm{e}+002$

$3.051 \mathrm{e}+002$

[K]
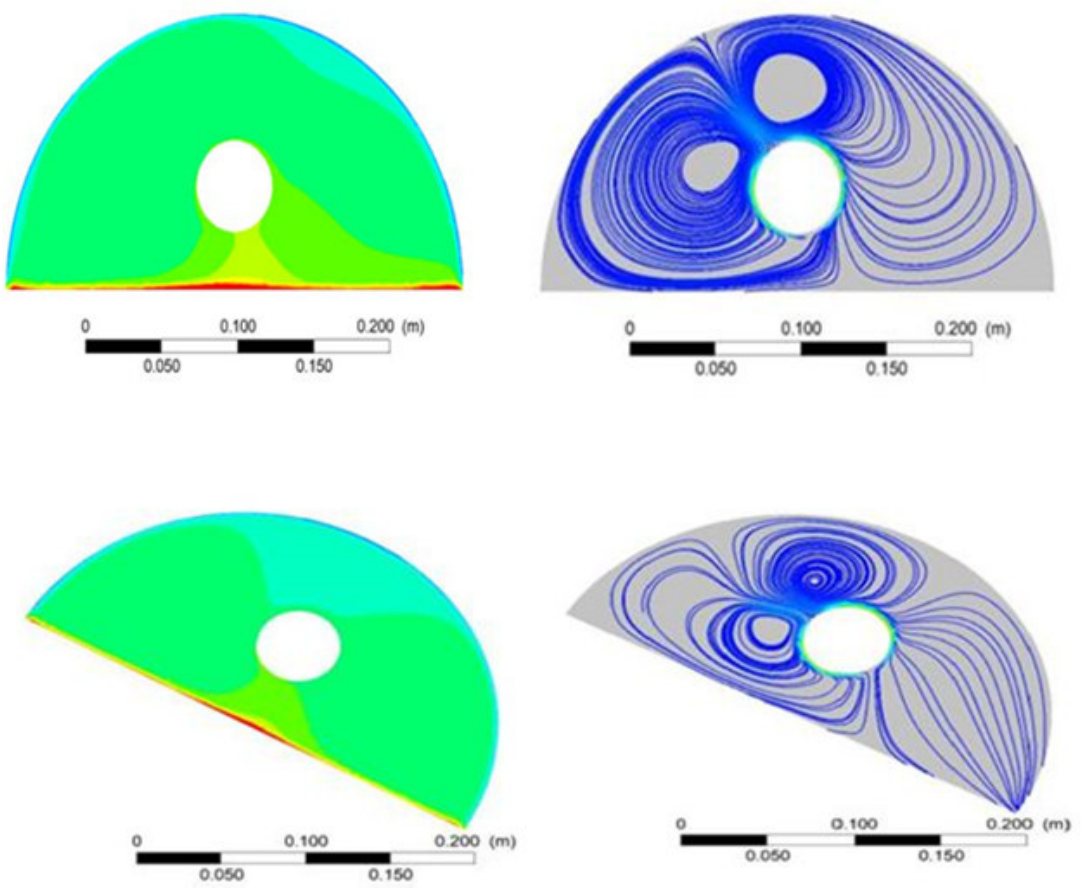

Velocity
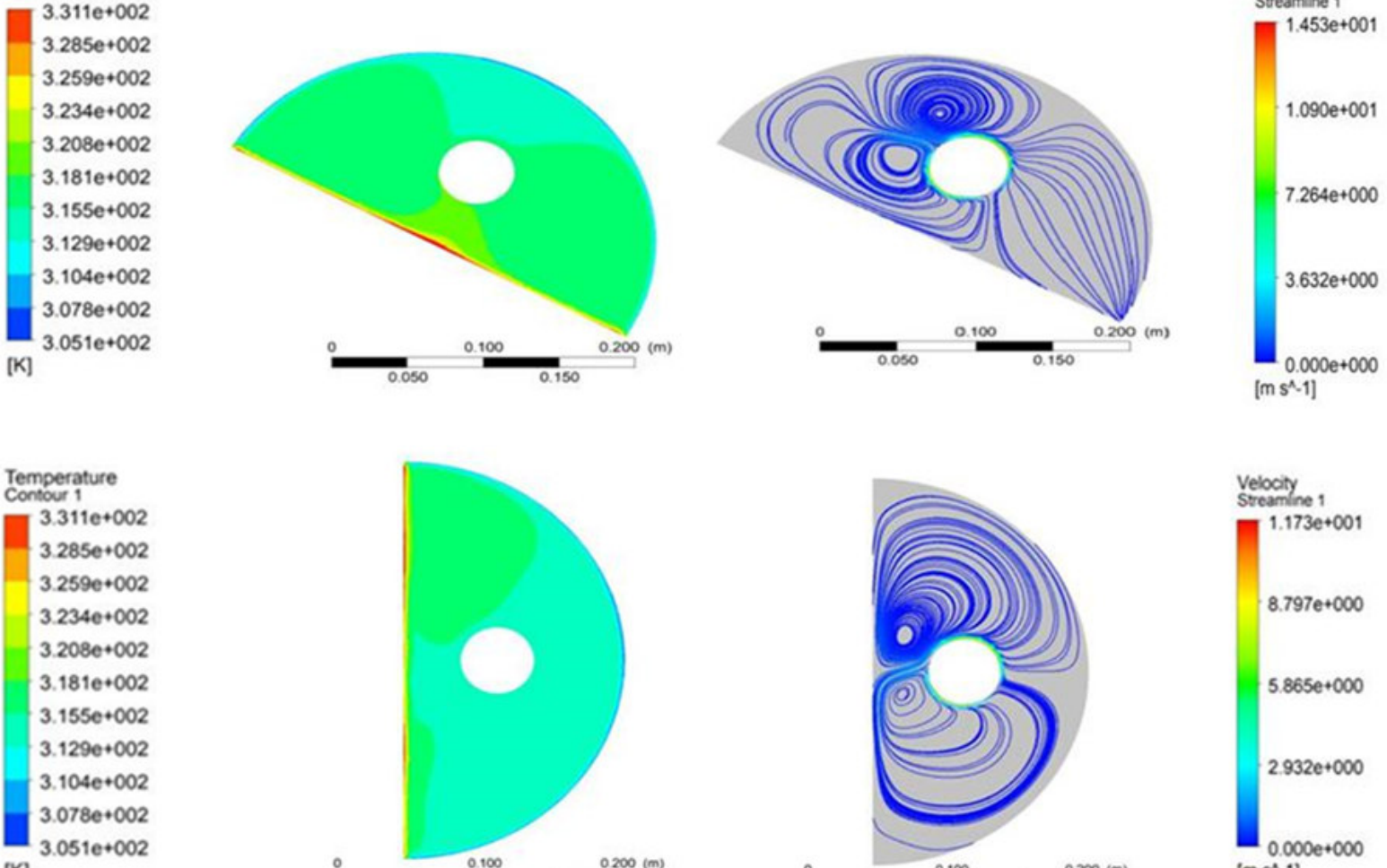

[K]
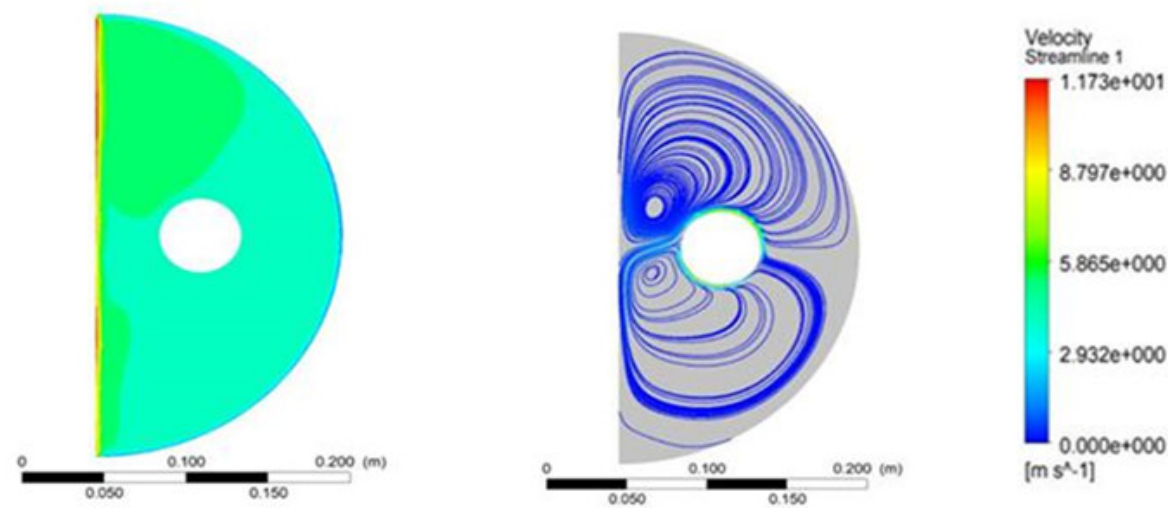

Fig.2.Temperature distribution and streamlines contours at q" $=290 \mathrm{w} / \mathrm{m} 2 \omega=62.8 \mathrm{rad} / \mathrm{s}$ and with inclination angle $\varnothing=0^{\circ}, 30^{\circ}, 90^{\circ}$ respectively. 


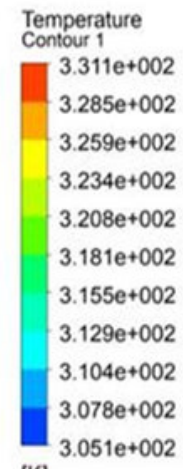

[K]

Temperature
Contour 1

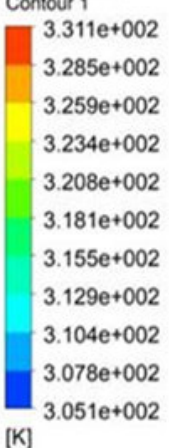

[K]

Temperature

$3.311 \mathrm{e}+002$

$3.285 \mathrm{e}+002$

$3.259 \mathrm{e}+002$

$3.234 \mathrm{e}+002$

$3.208 \mathrm{e}+002$

$3.181 \mathrm{e}+002$

$3.155 \mathrm{e}+002$

$3.129 \mathrm{e}+002$

$3.104 \mathrm{e}+002$

$3.078 \mathrm{e}+002$

$3.051 \mathrm{e}+002$

[K]
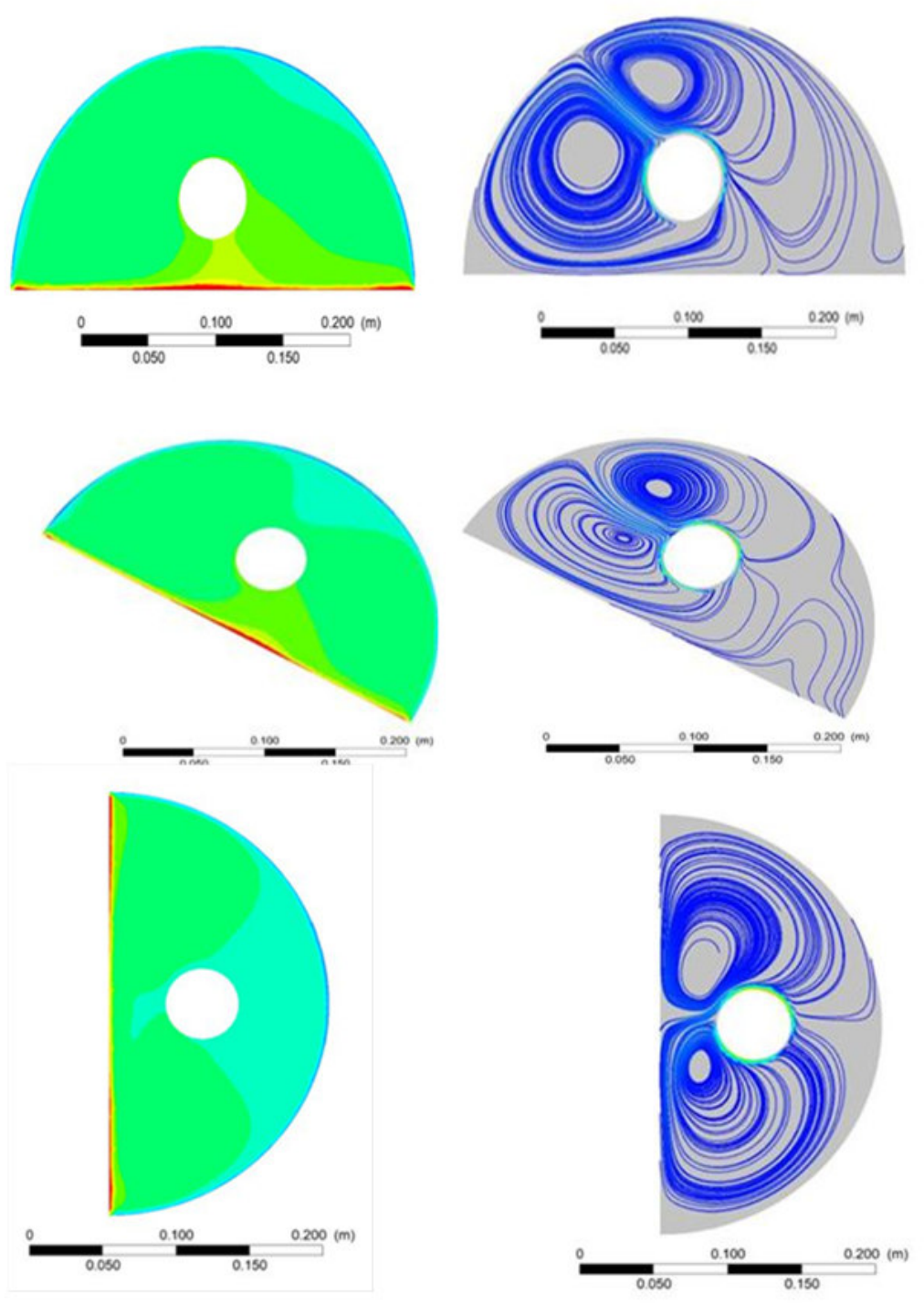

Fig.3.Temperature distribution and streamlines contours at q" $=290 \mathrm{w} / \mathrm{m} 2 \omega=83.73 \mathrm{rad} / \mathrm{s}$ and with inclination angle $\varnothing=0^{\circ}, 30^{\circ}, 90^{\circ}$ respectively. 


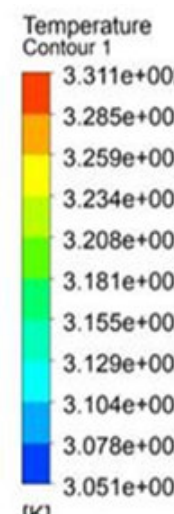

[K]

$051 \mathrm{e}+002$
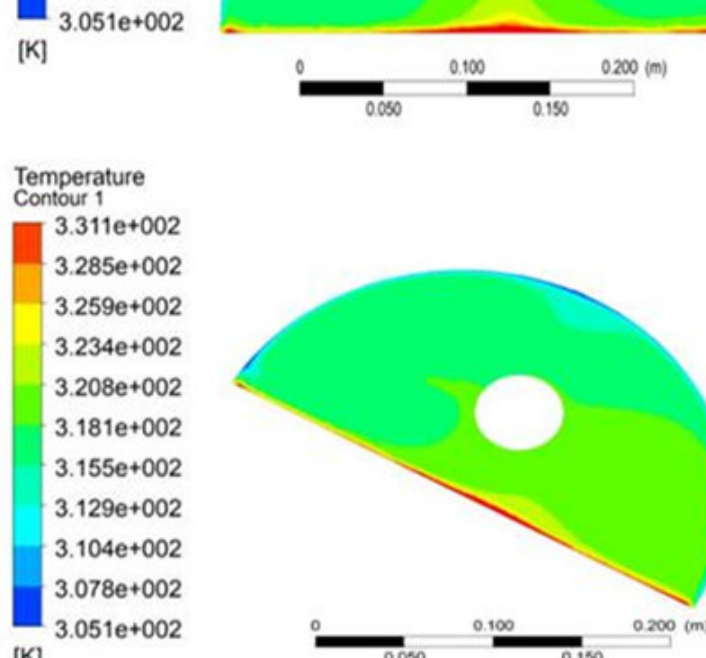

[K]
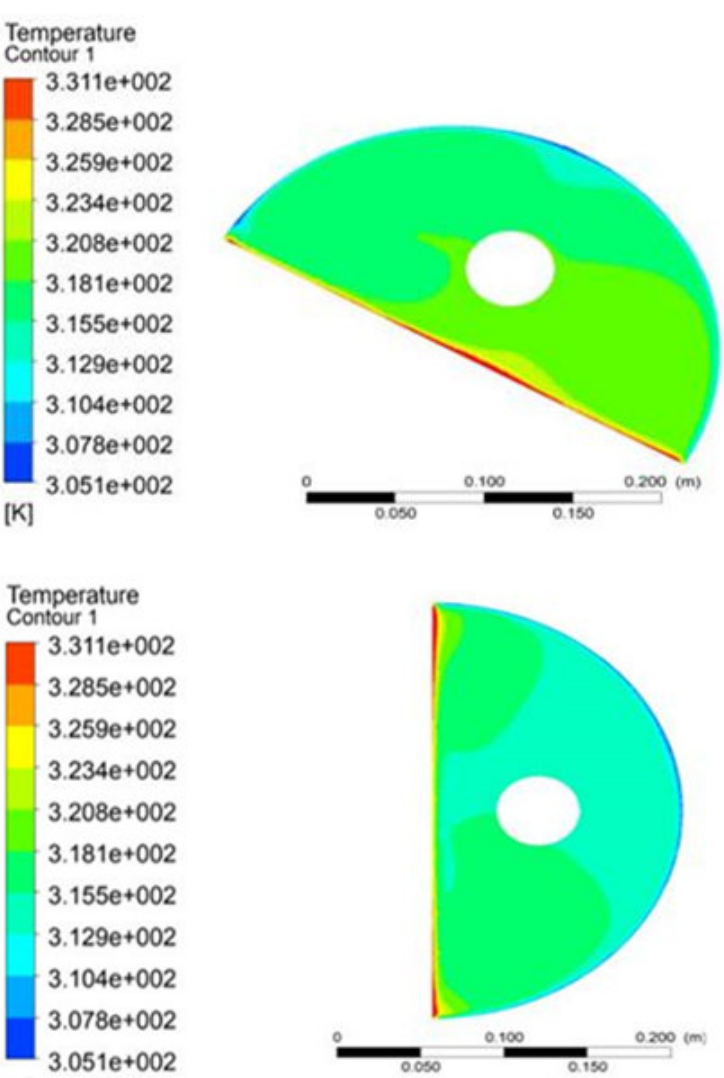
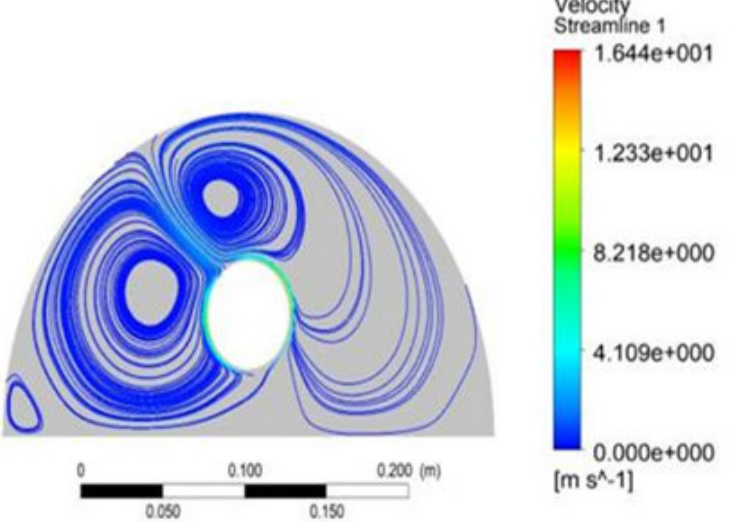

Velocity
Streamine 1 Streamine 1

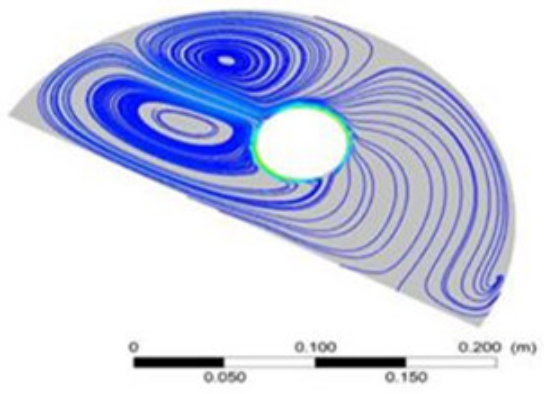

$1.553 \mathrm{e}+001$

$1.035 \mathrm{e}+001$

$5.176 \mathrm{e}+000$

$0.000 \mathrm{e}+000$ $\left[\mathrm{m} \mathrm{s}^{\wedge}-1\right]$

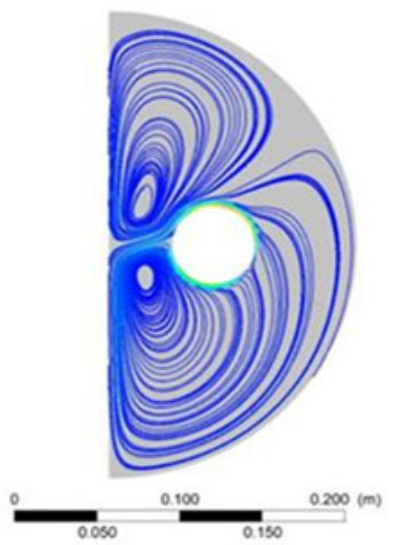

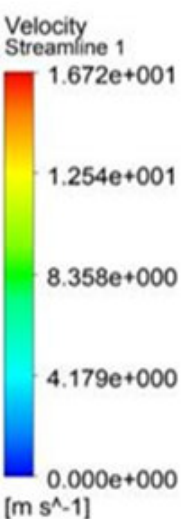

[K]

Fig.4.Temperature distribution and streamlines contours at q" $=290 \mathrm{w} / \mathrm{m} 2 \omega=89.5 \mathrm{rad} / \mathrm{s}$ and with inclination angle $\varnothing=0,30^{\circ}, 90^{\circ}$ respectively.

\subsection{Influence of Inclination Angle on Local Temperature}

Figure. 5 shows the impact of the angular velocity for on local temperature along the cross section of the heated wall at a fixed tilt angles .For this figure the value of heat flux $\mathrm{q}=290 \mathrm{w} / \mathrm{m} 2$ with constant angular velocity $\omega=62.8,83.73$ and $89.5 \mathrm{rad} / \mathrm{sand}$ different tilt angle. Fig.6 shows the distribution of temperature along the cross section of the hot surface at the distance $\mathrm{z}=0.375 \mathrm{~m}$. At the angular velocity $62.8 \mathrm{rad} / \mathrm{s}$ it can be noticed from the figure that temperature values are few at the two extremes of the hot surface near the curved surface, but increases at most angles of the tendency to reach the highest value in the middle of the area at below the rotary cylinder except at angle 90 we note the opposite, Its lowest value at the middle, this is due to the variation in the direction of convection currents formed on the hot surface with the flow direction. For angle 90, convection currents are parallel to the hot surface and opposite to the flow direction where they have little effect on flow. When increasing the angular velocity to the 83.73 and $89.5 \mathrm{rad} / \mathrm{s}$ it can be observed from this figure that the distribution of temperature is the same at angular velocity 62.8 a except for a small increase in their values due to increase the impact of forced convection, which in turn affects the gradient of fluid density in different areas 
along the hot surface.
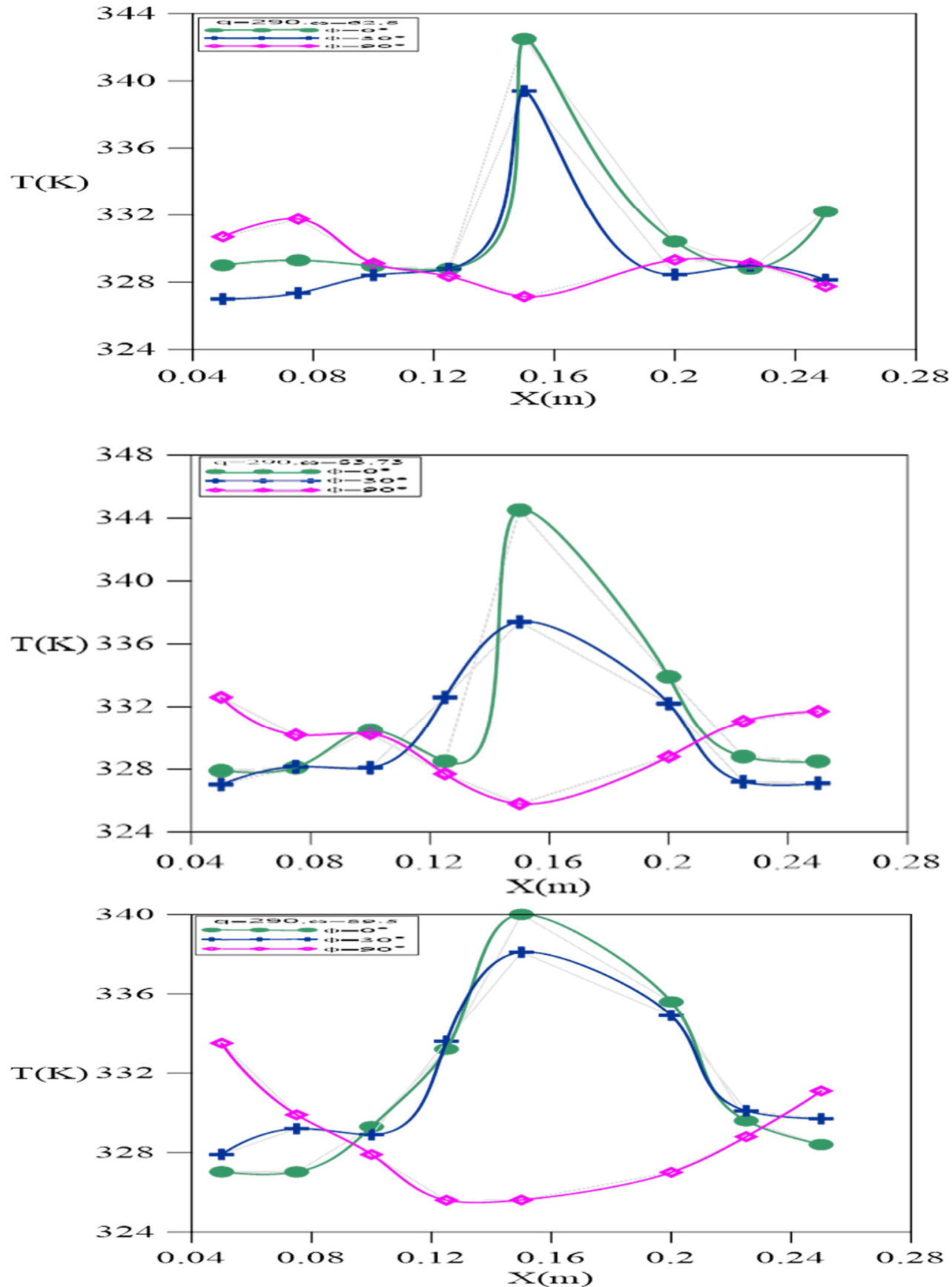

Fig.5. Temperature distribution along bottom wall at heat flux $290 \mathrm{w} / \mathrm{m} 2$ and different $\varnothing$ and $\omega=62.8,83.73$ and $89.5 \mathrm{rad} / \mathrm{s}$ respectively.

\subsection{Influence of Angular Velocity on Local Temperature}

Figure. 6 shows the influence of the angular velocity of the cylinder on the local temperature distribution along the bottom wall at a constant angles.For this figure the value of the heat flux $q=290 \mathrm{w} / \mathrm{m} 2$ with different angular velocity $\omega=62.8,83.73$ and $89.5 \mathrm{rad} / \mathrm{s}$. It can be observed that for the angle $\varnothing=0^{\circ}$, the temperature of the surface is high and that the distribution of the temperature will along the cross-section of the hot surface where we observe a small rise in the temperature of the area under the rotary cylinder due to the decreasing the resistance of heat transfer in the area narrow under the cylinder. At inclination angle $30^{\circ}$ shows the two temperature curves approaching along the heated wall. Shows in fixed tilt angle $90^{\circ}$ that the distribution of temperature at these angles depends on the angular velocity because of the difference in the convection cell in the intensity, that caused a remarkable changing in the temperature distribution. 

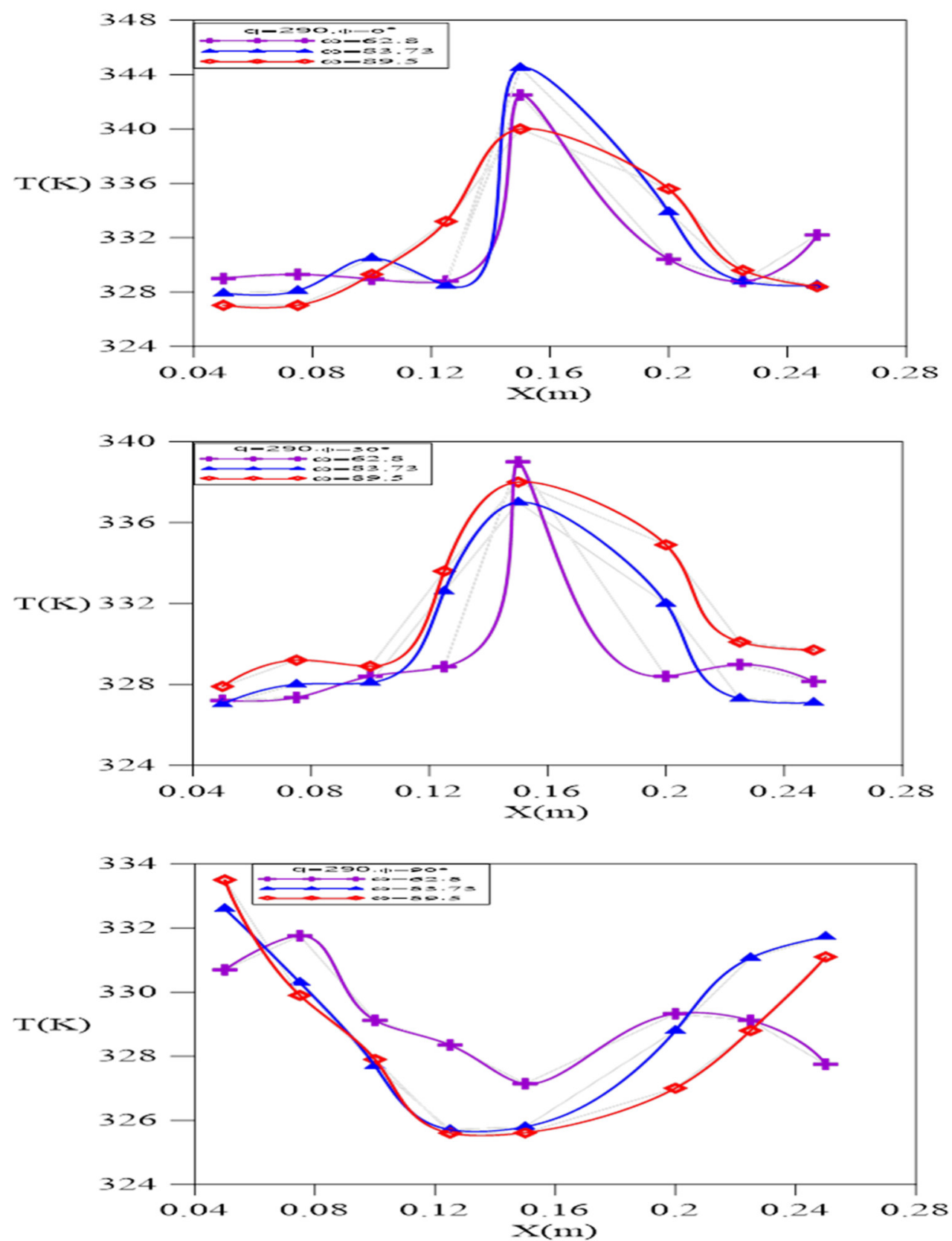

Fig.6. Temperature distribution along bottom wall at heat flux $\mathrm{q}^{\prime \prime}=290 \mathrm{w} / \mathrm{m} 2$ with different $\omega$ and $\varnothing=0^{\circ}, 30^{\circ}$ and $90^{\circ}$ respectively.

\subsection{Nusselt number}

Theoretical studied carried out with assist of local Nusselt number distribution along the lower wall of cavity. In this section, a series of runs with using value of heat flux were performed in the and theoretical results of the current work. Theoretical results introduce figures that illustrate the influence of inclination angle and angular velocity on heat transfer profile. Figure. 7 presents local Nusset number for heat flux $290 \mathrm{w} / \mathrm{m} 2$ and angular velocity $\omega=62.8,83.73$ and $89.5 \mathrm{rad} / \mathrm{s}$ respectively. Shows local Nusselt number which decreases in the midpoint of a the heated surface at the angles $0^{\circ}$ and $30^{\circ}$ due to increasing the resistance to flow in this area. The trend of the curve of local Nusselt number for angle $90 \mathrm{o}$ is give the opposite shape when compared with angles $0{ }^{\circ}$ and $30^{\circ}$ due to the direction of the flow is opposing the buoyancy effect. With increasing angular velocity to 83.73 , the numerical value of Nusselt was increased. Also for angle $60^{\circ}$ and with higher angular velocity $89.5 \mathrm{rad} / \mathrm{s}$, it can be seen that the decreasing in local Nusselt number and It can be noticeable the maximum peak subsist at $\mathrm{x}=0.08 \mathrm{~m}$ and the minimum peak at $\mathrm{x}=0.12 \mathrm{~m}$.

Figures .8show the variation of local Nusselt number along the cross section of the hot wall with influence of the angular velocity. This figure illustrates the local of Nusselt number for heat flux $290 \mathrm{w} / \mathrm{m} 2$, tilt angles 
$0^{\circ}, 30^{\circ}$ with various angular velocity. For the angle $0^{\circ}$ the increases angular velocity lead to increase Nusselt number because the impact of forced convection. At $\varnothing=30^{\circ}$ the decreasing of local Nusseltnumber can be observed in the midpoint with increasing the angular velocity due to a deep variaton of fluid temperature making high temperature difference between the hot wall and the fluid. For $\varnothing=90^{\circ}$ curves have opposite performance compared to other tilt angles because the two vortices are generated at upper and down portion of cavity is caused that to low temperature in the middle of the area.
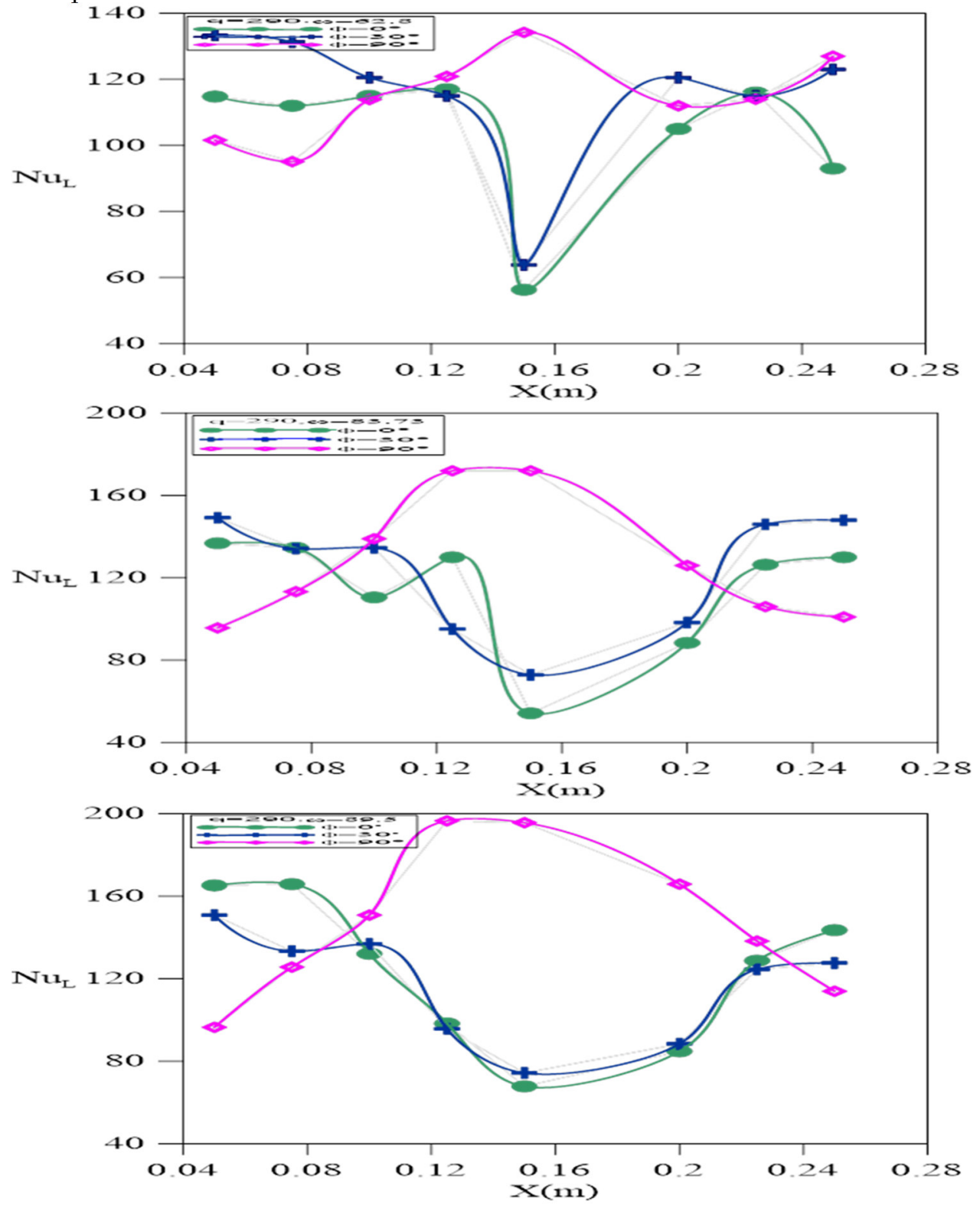

Fig.7.Variation of local Nusselt number along the bottom wall at heat flux $q=290 \mathrm{w} / \mathrm{m} 2$ with different $\varnothing$ and $\omega=62.8,83.73$ and $89.5 \mathrm{rad} / \mathrm{s}$ respectively. 

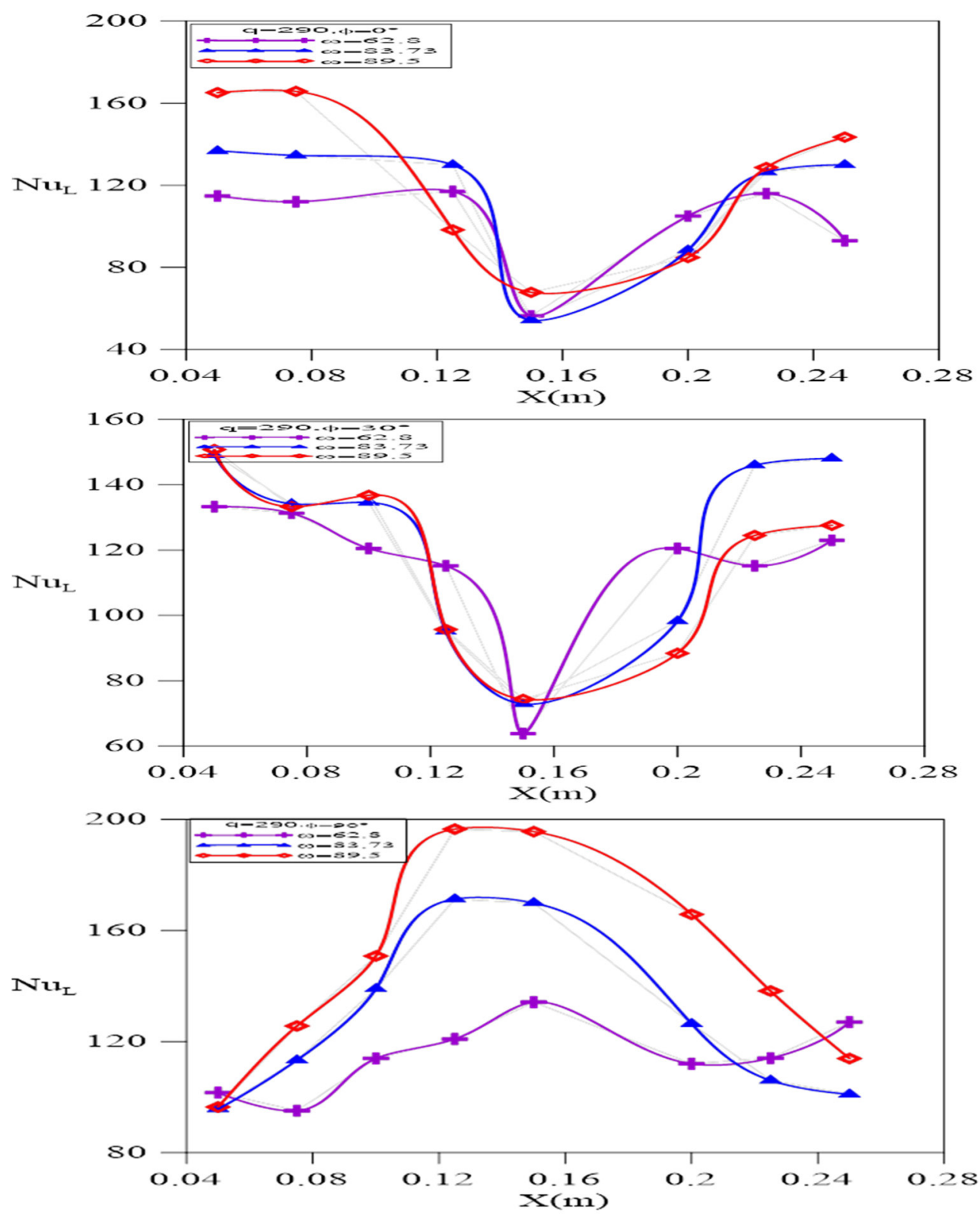

Fig.8.Variation of local Nusselt number along the bottom wall at heat flux $\mathrm{q}=290 \mathrm{w} / \mathrm{m} 2$ with different $\omega$ and $\varnothing=$ $0^{\circ}, 30^{\circ}$ and $90^{\circ}$ respectively

\section{Conclusion}

mixed convection heat transfer about adiabatic rotating cylinder within a semi circle cavity is studied at different angular velocity and various inclination angle.

1- The numerical results indicated that the convection heat transfer occurs is increasing with increasing the Rayleigh number

2- At angular velocity equal zero that means stationary cylinder show that the temperature contours are symmetrical around the midst of the cavity.

3 - At case stationary cylinder observes that the vortex movement unmoved by of inclination angle for placements at $\varnothing=0^{\circ}, 30^{\circ}$ and $90^{\circ}$.

\section{Reference}

[1] Srinivasan, J. (1993). Solar pond technology. Sadhana, 18(1), 39-55.

[2] Billah, M. M., Rahman, M. M., Saidur, R., \& Hasanuzzaman, M. (2011). Simulation of mhd mixed convection heat transfer enhancement in a double lid-driven obstructed enclosure. International Journal of Mechanical and Materials Engineering, 6(1), 18-30.

[4] Karimi, F., Xu, H., Wang, Z., Yang, M., \& Zhang, Y. (2016). Numerical simulation of steady mixed convection 
around two heated circular cylinders in a square enclosure. Heat Transfer Engineering, 37(1), 64-75.

[3] Alam, M., Kamruzzaman, Ahsan, F., \& Hasan, M. N. (2016, July). Mixed convection heat transfer inside a differentially heated square enclosure in presence of a rotating heat conducting cylinder. In AIP Conference Proceedings (Vol. 1754, No. 1, p. 050035). AIP Publishing.

[5]Park, Y. G., Yoon, H. S., \& Ha, M. Y. (2012). Natural convection in square enclosure with hot and cold cylinders at different vertical locations. International Journal of Heat and Mass Transfer, 55(25-26), 79117925.

[6]Chatterjee, D., \& Mishra, R. (2018). Numerical investigation of transient magnetohydrodynamic mixed convection in a ventilated cavity containing two heated circular cylinders. Heat Transfer Engineering, 39(12), 1052-1066.

[7] Alhattab, T. A. H., Alsaegh, A. S. N., \& Alinnawi, F. H. A. (2011). Natural Convection in Equilateral Triangular Enclosure for Three Situations with Heating Element Applied at Different Positions on the Inclined Side Wall. Iraqi journal of mechanical and material engineering, 11(4), 619-636.

[8] Mushatet, K. S., \& Mehdi, Q. S. (2009). Numerical Investigation of Turbulent Natural Convection in an Inclined Square Enclosure. Journal of Engineering, 15(1), 3466-3481..

[9]Singh, S., \& Bhargava, R. (2014). Numerical study of natural convection within a wavy enclosure using Meshfree approach: effect of corner heating. The Scientific World Journal, 2014.

[10]Rincón-Casado, A., Sánchez de la Flor, F. J., Chacón Vera, E., \& Sánchez Ramos, J. (2017). New natural convection heat transfer correlations in enclosures for building performance simulation. Engineering Applications of Computational Fluid Mechanics, 11(1), 340-356.

[11] Cable, M. (2009). An evaluation of turbulence models for the numerical study of forced and natural convective flow in Atria(Doctoral dissertation, Queen's University).

[12] Theraja, B. L., \& Sedha, R. S. (2006). TB Of Electrical Technology Vol Iv (ME) (Vol. 4). S. Chand. 